\title{
The evolution of microbial biofilms on sandy rocks in aquifers with anthropogenic pollution
}

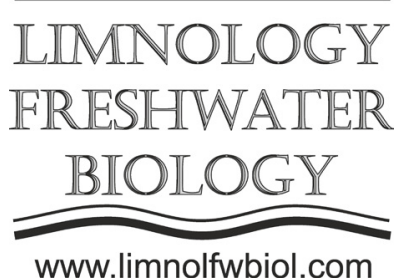

\author{
Popova N.M.*, Safonov A.V. \\ Frumkin Institute of Physical Chemistry and Electrochemistry RAS (IPCE RAS), Leninsky Prospekt, 31/4, Moscow, 119071, Russia
}

\begin{abstract}
Organic and inorganic pollution can increase microbial biofilms formation on sandy rocks of the upper aquifers. The activity of microorganisms in biofilms can cause very effective remediation of the aquifers, and their study is important in the framework of groundwater bioremediation. Chemical and taxonomic transformation of the biofilms microbial community of upper aquifers nearby radioactive waste and uranium sludge repositories polluted with nitrates, chromate and uranyl ions after single glucose stimulation was studied in the work.
\end{abstract}

Keywords: Biofilms, aquifer, nitrates, radionuclides.

\section{Introduction}

Industrial pollution of groundwater with organic substances, anions of mineral acids, heavy metals and radionuclides can intensify the development of microbial biofilms in upper aquifers host rock. The biogeochemical activity of microbial biofilms can significantly reduce the uncontrolled migration of pollutants, and the use of a number of in situ methods to stimulate this process will minimize the risks of toxic compounds entering water to open ecosystems. The study of biofilm formation and evolution, both chemical and taxonomic, is an important part of the preparation for upper aquifers remediation near radioactive waste and uranium sludge storage facilities, with a high level of contamination with acid anions (nitrates, sulfates), radionuclides and heavy metals. Sampling was carried out on the territory of Rosatom facilities «SCC», «AECC», «ECP» and «NZHK». In this work, we studied the microbial communities development in water and rock samples of the upper aquifers in dynamic.

\section{Material and methods}

Here we used one time glucose stimulation of water and rocks samples in $15 \mathrm{ml}$ penicillin vials. Than every 5 days we sampled an aliquots and then analysed them by following methods: visualization by electron and laser confocal scanning microscopy using the Imaris software package, determination of total organic matter by wet burning according to Tyurin, determination of the respiratory activity of microbial cells of biofilm and plankton cells (MTT test), determination of the taxonomic composition of communities by pyrosequencing of 16s rRNA genes, analysis of the chemical composition of biofilms determination of proteins (according to Lowry), sugars (according to Dubois) and visual confirmation by staining with FITC and WGA lectin.

\section{Results and discussion}

Visual studies of daily samples showed that biofilm growth began on the first day; the most active formation of the EPS matrix of biofilms occurs in the period from 8 to 15 days, and at this time a variety of epilithic forms of biofilms can be observed (Fig. 1).

Respiratory activity of developing biofilms of all studied objects is higher on the rock than in the supernatant. An increase in the activity of plankton cells in samples with a long period of biofilm existence on individual rocks can be associated with the resettlement stage of biofilms (Petrova and Sauer, 2016).

The analysis of total organic matter revealed that the biofilm produces the greatest amount of organic carbon in the period of 20 days of its existence; subsequently, the amount of organics on the rock decreases due to a decrease in the activity of microorganisms (Fig. 2). Elemental analysis on a CHN analyzer confirms the mass \% concentration of nitrogen $(2 \pm 0.5 \%)$, carbon $(8 \pm 0.65 \%)$.

As a result of the application of high-performance sequencing of the V3 - V4 region of the 16S rRNA gene, libraries of gene fragments were obtained for samples from two biofilms formed on the sandy rock SCC B-2/32 on the 7th and 21st days after glucose stimulation. Representatives of the Comamonadaceae family dominated in both samples: Comamonas 

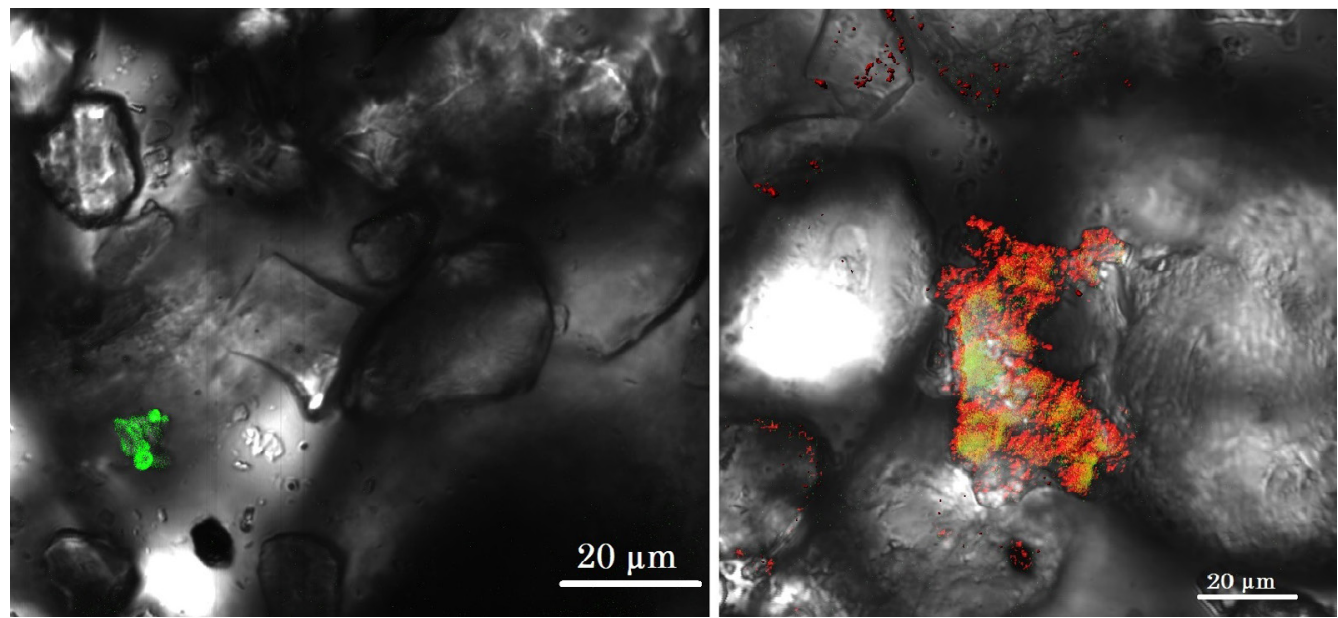

Fig.1. Sandy rock before and after biofilm formation

(68.99-34.6\%), Delftia (1\%), Hydrogenophaga (0.5\%), capable of denitrification, hydrogen consumption, sulfate reduction and reduction of Fe (III) compounds, organochlorine compounds and other complex organic substances under anaerobic conditions (Maamar et al., 2015). In addition, representatives of the genus Paenibacillus (27\%) and Thermomonas (28.4 - 36.6\%) was shown.

\section{Conclusions}

During biofilm development, fouling of rock particles and the formation of epilithic forms, the biofilm reaches its maximum by $15-20$ days, then begins gradual degradation of the biofilm matrix. Communities developing on rocks with a high content of clay fraction are exceptions.

There is a change in the chemical composition of the matrix, which is correlated with the dynamics of the development of biofilms.
Metabolic heterogeneous microbial community able to form microbial biofilms and ensure the restoration of chromate and uranyl ions to low mobility forms and remove nitrate ions was found in rock samples. The succession of the microbial community in the development of biofilms was studied. At the first stages, predominantly aerobic bacteria developed, then microaerophilic-anaerobic microorganisms dominated.

\section{References}

Petrova O.E., Sauer K. 2016. Escaping the biofilm in more than one way: desorption, detachment or dispersion. Current Opinion in Microbiology 30: 67-78. DOI: 10.1016/ j.mib.2016.01.004

Maamar B.S., Aquilina L., Quasier A. et al. 2015. Groundwater isolation governs chemistry and microbial community structure along hydrologic flowpaths. Frontiers in Microbiology 6. DOI: 10.3389 / fmicb.2015.01.01457

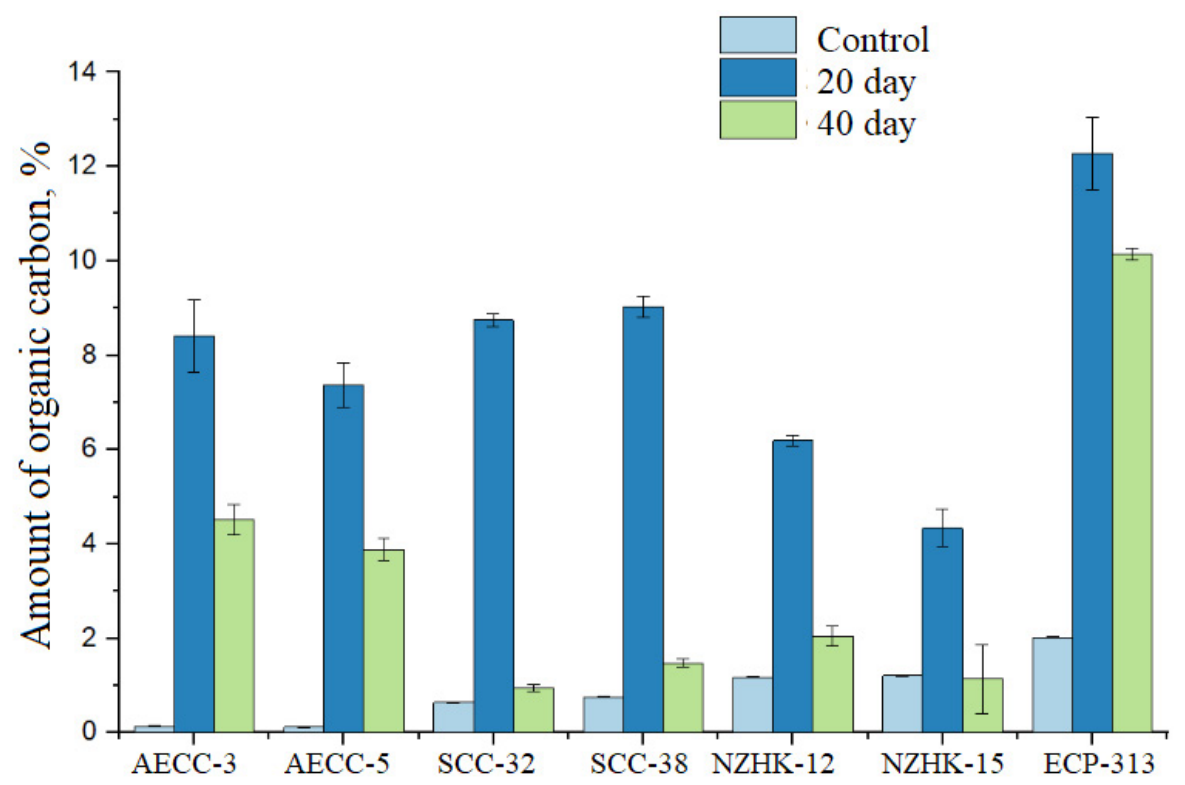

Fig.2. Organic carbon production during cultivation 